\title{
表面物理化学
}

吴凯

北京大学化学与分子工程学院, 北京 100871

\section{Surface Physical Chemistry}

\section{WU Kai}

College of Chemistry and Molecular Engineering, Peking University, Beijing 100871, P. R. China.

Email: kaiwu@pku.edu.cn

表面物理化学是物理化学中的重要分支之 一, 主要涵盖表面化学与物理。表面物理化学探究 固体表面物理化学过程的基本原理, 在多相催化、 电化学、能源化学以及纳米科技等领域具有广泛 的应用。例如, 2007 年的诺贝尔化学奖得主 G. Ertl 教授曾指出 “整个纳米技术领域实际上就是由表 面反应控制的” ${ }^{1}$ 。随着现代表面科学技术的快速 发展和理论化学的进步, 人们现在可以在原子和 分子层次上揭示表面物理化学过程。

表面物理化学的重要研究方向之一是表面催 化。催化的核心使命是以更加经济、高效和环境友 好的方式将原料转化为具有高附加值的化工品和 燃料等, 涉及化学、食品、医药、汽车以及石油化 工等重大支柱性产业, 在人类文明进步和经济发 展中占有举足轻重的地位。催化一般分为多相与 均相催化。在能源和化工等规模化化学工业生产 中, $90 \%$ 以上的过程涉及多相催化 ${ }^{2}$ 。

多相催化研究经历了长期的历史发展过程, 不断汲取和借鉴化学及相关学科中发展起来的新 的科学原理、方法和技术来探索催化过程中的基 本化学和物理性质 3 。多相催化过程主要在催化剂 固体表面或界面上发生, 其研究通常分为两部分: 从固体表面的视角, 包括固体表界面组成、结构、 电子性质和能量等; 从反应分子的视角, 包括分子 结构变迁与化学键的断裂和形成。催化化学的研 究关键是揭示催化转化过程中的构-效关系, 即催 化体系的结构与性能之间的关系 4 。

多相催化的研究极为复杂, 需要多学科的综 合交叉, 涉及化学与物理, 表面与反应工程, 理论
与实验, 谱学与动力学, 以及纳米科技与材料科学 等等。工业催化剂是复杂的材料, 其物理和化学特 性往往依赖于其组成、结构、形态和粒子形貌等参 数。因此, 多相催化基本原理的发展大多依赖于对 理想反应器中、温和条件下的模型催化剂和模型 催化反应体系的研究, 而不是实际工业生产条件 下、混合进料体系以及复杂反应器中的商用催化 剂的催化性能数据 5 。但这些模型催化体系的研究 推动了催化科学的发展, 引导着人们开发新的催 化剂和催化过程 6 。

实验室中的催化研究通常涉及实用催化剂的 设计制备和催化反应性能。前者涉及固体表面的 活性位点(或活性中心), 包括活性位点的原子结构 与组分及其在反应条件下的动态变化; 后者包括 过程条件下目标产物的选择性、反应速率、催化剂 寿命以及活性位点的可接触性等。

要揭示这些催化基本原理, 原子或分子层次 的研究方法与技术是必不可少的。催化本质上是 动力学过程, 无论是在动力学数据测量还是活性 位点的结构与成份变化、反应机理以及催化过程 中体系的能量变化的研究中, 超高空间和时间分 辨的检测技术均发挥了重要作用, 这涉及到表面 物理化学的方方面面 7 。

然而, 由于技术方法的本身限制, 催化原理的 研究常常缺乏翔实的可用数据, 如催化反应机理, 反应活性中间物种的检测、基元反应步骤的确定 以及反应过程的能量变化等。在大量实验数据的 基础上, 人们也发展了诸多理论与计算方法来分 析这些数据、建立模型、探讨催化机理与规律 ${ }^{8}$ 。 
因此, 实验与理论的紧密结合对于催化研究至关 重要。

多相催化中的表面物理化学研究涵盖了催化 剂的设计与制备、催化反应性能、表面活性位点、 反应机理和能量变化等方面开展实验与理论相结 合的研究。特别地, 人们期望能够综合利用超高空 间和时间分辨的表面科学技术与方法, 在原子和 分子水平上对实用和模型催化剂表面的物理和化 学过程进行详细的研究, 为新型催化体系的研发 提供科学基础。

在多相催化模型研究中, 人们通常关注以下 关键科学问题: (1) 催化剂表面活性位点的结构与 动态演化, 涉及金属颗粒的尺寸与形貌, 以及电子 性质的调控与催化性能的关联。不同晶面的催化 活性差异, 金属-载体界面结构、电荷转移与催化 性能, 以及反应过程中表面组成与相变等导致的 活性位点变化。(2) 表面位点与反应分子间的作 用, 包括反应分子诱导的结构变化, 位点-分子和 分子间的电荷转移与能量传递等。

本特刊专辑邀请了国内部分从事表面物理化 学研究的学者团队, 介绍他们近年来在表面物理 化学若干方面的研究进展和总结。主要分为三类: 表面单分子性质的研究; 规整单晶表面上小分子 的吸附、活化与反应的研究; 模型催化剂制备及其 催化性质的研究。

下面对这些研究和总结进行扼要介绍。

\section{(1) 表面单分子性质的研究}

王兵研究小组 ${ }^{9}$ 在 $\mathrm{Au}(111)$ 表面制备高质量的 $\mathrm{N} @ \mathrm{C}_{60}$ 分子, 并利用扫描隧道显微镜(STM)研究了 表面上该分子的结构及电子态。

\section{(2) 规整单晶表面上小分子的吸附、活化与反应 的研究}

陈明树研究小组 10 利用高分辨电子能量损失 谱(HREELS)、俄歇电子能谱(AES)和低能电子衍 射(LEED)研究了镍单晶表面氧物种及 $\mathrm{CO}$ 与 $\mathrm{O}_{2}$ 的 共吸附。

刘志研究小组 ${ }^{11}$ 及其合作者利用近常压光电 子能谱 (APXPS) 和 STM 原位研究了 $\mathrm{H}_{2}$ 在 $\mathrm{ZnO}(10 \overline{1} 0)$ 表面上的活化和解离。

许维研究小组 ${ }^{12}$ 综述了近年来人们利用 STM 和密度泛函理论(DFT)计算方法研究超高真空环 境下碱基及其衍生物与碱金属、碱土金属和过渡 金属的相互作用, 总结了碱基与金属的作用位点 及反应发生的机理。

\section{(3) 模型催化剂制备及其催化性质的研究}

杨帆研究团队 13 利用 $\mathrm{NO}_{2}$ 或 $\mathrm{O}_{2}$ 作为氧化剂,
研究了氧化锌在 $\mathrm{Au}(111)$ 和 $\mathrm{Cu}(111)$ 上的生长和结 构, 并进行了详细表征。

朱俊发研究小组 ${ }^{14}$ 研究了 $\mathrm{CeO}_{2}(111)$, 部分还 原的 $\mathrm{CeO}_{2-x}(111)(0<x<0.5)$ 以及 $\mathrm{Ca}$ 掺杂的 $\mathrm{CeO}_{2}$ 模型催化剂的形貌、电子结构以及它们与 $\mathrm{CO}_{2}$ 分 子间的相互作用。

邵翔研究小组 ${ }^{15}$ 在 $\mathrm{Au}(111)$ 表面上制备了由 蜜勒胺分子形成的具有周期性孔道结构的自组装 薄膜, 在室温下暴露 $\mathrm{CO}$ 气体, 在表面上成功制备 得到稳定的 $\mathrm{Au}$ 单原子。

陆军岭研究小组 16 综述了原子层沉积(ALD) 技术方法在负载型单金属和双金属催化剂精细设 计中的进展, 特别是单原子和双原子结构金属催 化剂的制备。

王显研究小组 17 合成了一种直接生长在泡沫 镍基底的 $\mathrm{NiS}-\mathrm{Ni}_{3} \mathrm{~S}_{2}$ 树状异质结阵列, 在电解水的 阳极反应中表现出高效电催化性能和超高稳定性 能。

希望《物理化学学报》的广大读者能够喜欢这 些文章, 阅读愉快, 并从中得到启迪。

\section{References}

(1) Ertl, G. Reactions at Solid Surfaces; John Wiley \& Sons, Inc.: Hoboken, New Jersey, 2008.

(2) Ertl, G.; Knözinger, H.; Schüth, F.; Weitkamp J. (Eds.) Handbook of Heterogenous Catalysis (2nd Ed.); Wiley-VCH, Verlag GmbH: Weinheim, Germany, 2008.

(3) Zecchina, A.; Califano, S. The Development of Catalysis - A History of Key Processes and Persona in Catalytic Science and Technology; John Wiley \& Sons, Inc.: Hoboken, New Jersey, 2017.

(4) Somorjai, G. A.; Li, Y. Introduction to Surface Chemistry and Catalysis; John Wiley \& Sons, Inc.: Hoboken, New Jersey, 2010.

(5) Thomas, J. M.; Thomas, W. J. Principles and Practice of Heterogeneous Catalysis; VCH Verlagsgesellschaft mbH: Weinheim, Germany, 1997.

(6) Frenken, J.; Groot, I. (Eds.) Operando Research in Heterogeneous Catalysis; Springer International Publishing AG: Cham, Switzerland, 2017.

(7) Park, J. Y. (Ed.) Current Trends of Surface Science and Catalysis; Springer Science + Business Media: New York, 2014.

(8) Gro $\beta$, A. Theoretical Surface Science-A Microscopic Perspective; Springer-Verlag: Berlin, Germany, 2009.

(9) Zhao, Y. L.; Wang, B. Acta Phys. -Chim. Sin. 2018, 34 (12), 1312. [赵烨梁, 王兵. 物理化学学报, 2018, 34 (12), 1312.] doi: 10.3866/PKU.WHXB201803011 (10) Duan, Y.; Chen, M. S.; Wan, H. L. Acta Phys. -Chim. Sin. 2018, 34 
(12), 1358. [段园, 陈明树, 万惠霖. 物理化学学报, 2018, 34 (12), 1358.] doi: 10.3866/PKU.WHXB201803071

(11) Liu, Q.; Han, Y.; Cao, Y. J.; Li, X. B.; Huang, W. G.; Yu, Y.; Yang, F.; Bao, X. H.; Li, Y. M.; Liu, Z. Acta Phys. -Chim. Sin. 2018, 34 (12), 1366. [刘强, 韩永, 曹云君, 李小宝, 黄武根, 余毅, 杨帆, 包信 和, 李毅敏, 刘志. 物理化学学报, 2018, 34 (12), 1366.] doi: 10.3866/PKU.WHXB201804161

(12) Wang, X. Y.; Xie, L.; Ding, Y. Q.; Yao, X. Y.; Zhang, C.; Kong, H. H.; Wang, L. K.; Xu, W. Acta Phys. -Chim. Sin. 2018, 34 (12), 1321. [王 心怡, 谢否, 丁元琪, 姚心仪, 张弛, 孔惠慧, 王利坤, 许维. 物理 化学学报, 2018, 34 (12), 1321.] doi: 10.3866/PKU.WHXB201802081

(13) Zhao, X. F.; Chen, H.; Wu, H.; Wang, R.; Cui, Y.; Fu, Q.; Yang, F.; Bao, X. H. Acta Phys. -Chim. Sin. 2018, 34 (12), 1373. [赵新飞, 陈 浩, 吴昊, 王睿, 崔义, 傅强, 杨帆, 包信和. 物理化学学报, 2018,
34 (12), 1373.] doi: 10.3866/PKU.WHXB201804131

(14) Wang, Y.; Li, X.; Hu, S. W.; Xu, Q.; Ju, H. X.; Zhu, J. F. Acta Phys. -Chim. Sin. 2018, 34 (12), 1381. [王岩, 李雄, 胡善玮, 徐倩, 鞠焕釒金, 朱俊发. 物理化学学报, 2018, 34 (12), 1381.] doi: 10.3866/PKU.WHXB201804092

(15) Huang, L. L.; Shao, X. Acta Phys. -Chim. Sin. 2018, 34 (12), 1390. [黄丽丽, 邵翔. 物理化学学报, 2018, 34 (12), 1390.] doi: 10.3866/PKU.WHXB201804191

(16) Wang, H. W.; Lu, J. L. Acta Phys. -Chim. Sin. 2018, 34 (12), 1334. [王恒伟, 陆军岭. 物理化学学报, 2018, 34 (12), 1334.] doi: 10.3866/PKU.WHXB201804201

(17) Luo, P.; Sun, F.; Deng, J.; Xu, H. T.; Zhang, H. J.; Wang, Y. Acta Phys. -Chim. Sin. 2018, 34 (12), 1397. [罗盼, 孙芳, 邓菊, 许海涛, 张慧娟, 王显. 物理化学学报, 2018, 34 (12), 1397.] doi: 10.3866/PKU.WHXB201804022 